\title{
Genetic Elements Associated With Antimicrobial Resistance Among Intestinal Bacteria
}

\author{
Bita Bakhshi ${ }^{1, *}$; Nazanin Eftekhari ${ }^{2}$; Mohammad Reza Pourshafie ${ }^{3}$ \\ ${ }^{1}$ Department of Bacteriology, Faculty of Medical Sciences, Tarbiat Modares University, Tehran, IR Iran \\ ${ }^{2}$ Department of Biology, Faculty of Basic Science, Science and Research Branch, Islamic Azad University, Tehran, IR Iran \\ 3 Department of Bacteriology, Pasteur Institute of Iran, Tehran, IR Iran \\ ${ }^{*}$ Corresponding author: Bita Bakhshi, Department of Bacteriology, Faculty of Medical Sciences, Tarbiat Modares University, Jalal-Ale-Ahmad Ave., Tehran, IR Iran. Tel: +98-2182884558, \\ Fax:+98-2182884555, E-mail: b.bakhshi@modares.ac.ir
}

Received: December 23, 2012; Revised: June 16, 2013; Accepted: July 11, 2013

\begin{abstract}
Background:Integrons are the major reasons of multidrug resistance(MDR)among enteropathogenic bacteria. Occurrence of horizontal gene transfer between integron-carrying microorganisms and other enteric bacteria may increase the rate of emergence of integronassociated antibiotic resistance.

Objectives: The objective of this study was to investigate class 1 integrons among members of enteropathogenic bacteria isolated from patients in Iran.

Materials and Methods: A total of 120 enteropathogenic bacterial isolates from diarrhoeal patients were included in this study. Identities of the isolates were investigated by biochemical tests and confirmed by genus or species specific PCRs. Antimicrobial susceptibility testing was performed according to the Clinical and Laboratory Standards Institute (CLSI) guidelines. Presence of class 1 integron among the isolates was investigated using primers specific for the integrase gene conserved region.

Results: The result of this study showed the highest resistance to trimethoprim and cotrimoxazole, especially in enteropathogenic Escherichia coli (EPEC) (100\%), Shigella sonnei (93.7\%) and Vibrio cholerae (95\%). The results showed that 16 (57.1\%) of the 28 EPEC, 9 (25\%) of the 36 Salmonella enterica, 32 of the $13(40.6 \%)$ Sh. sonnei, and only 1 (4.2\%) of the 24 V. cholerae isolate harbored class 1 integron.

Conclusions: The data obtained in the present study suggested that class 1 integrons are widely distributed among members of Enterobacteriaceae. The resistance patterns of our E. coli, S. sonnei, and S. enterica isolates were nearly identical, suggesting the same genetic elements involved in attainment of multi-drug resistance.
\end{abstract}

Keywords: Integrons; Drug Resistance; Antimicrobial Agents; Intestinal Bacteria

\section{Background}

Diarrhea occurs worldwide and is most commonly caused by enteropathogenic bacterial infections, killing annually around 2.2 million people globally, mostly children, in developing countries (1). Because of the increased resistance to most of the widely-used antibiotics, effective treatment has become more difficult in recent years. It has been shown that the resistance genes present on the plasmids and transposons of Gram-negative bacteria can be integrated into DNA elements called "integrons" (2). Integrons are the major reason of multidrug resistance (MDR) in enteropathogens (3).

Occurrence of horizontal gene transfer between integroncarrying microorganisms and other enteric bacteria may increase the emergence rate of integron-associated antibiotic resistance $(4,5)$. As the case for most other antibiotic-resistant pathogens, the spread of integron-carrying MDR enteropathogens has usually been detected during outbreaks of nosocomial infection $(4,6)$. Integrons can carry a variety of antibiotic resistance genes, inserted as cassettes (7). There are five different integron classes with different integrated gene cassettes, among which class 1 and 2 integrons are most commonly found within enteropathogens (8).

Integrons are common in nature, and class 1 integrase gene, intI1, is detected as the majority of integrons found in clinical isolates (7). This integron class is the most widely studied and defined. Integrons have been found among clinical and commensal isolates of both humans and animals. Even though more than 60 drug-resistance genes have been identified in class 1 integrons, it is becoming apparent that the common gene cassettes are those associated with aminoglycoside and trimethoprim resistance. Moreover, studies have clearly demonstrated swapping of these antibiotic resistance genes into integrons; therefore, presenting integrons as important tools in the rapid evolution and dissemination of antibiotic re sistance among Gramnegative microorganisms (9). 
Bakhshi B et al.

\section{Objectives}

The aim of this study was to identify class 1 integrons among members of enteropathogenic bacteria, including Salmonella enterica, Shigella sonnei, enteropathogenic E. coli (EPEC) and Vibrio cholerae strains, isolated from patients in Iran.

\section{Materials and Methods}

\subsection{Clinical Isolates}

A total of 120 enteropathogenic bacterial isolates from stool samples of diarrhoeal patients were included in this study (Table 1). The isolates were randomly selected from our bacterial collection, gathered during a 3-year period from Tehran province. Biochemical screening tests and serogrouping were performed to characterize the Sh. sonnei, S. enterica, EPEC and V. cholerae, and the identities of isolates were confirmed using primer sets, which specifically detected these enteropathogens as described elsewhere (Table 1).

\subsection{Antimicrobial Susceptibility Testing}

The antimicrobial agents were selected among those commonly carried by integrons. Antimicrobial susceptibility testing was performed using disc diffusion method according to the Clinical and Laboratory Standards Institute (CLSI) guidelines for each bacterial species $(15,16)$, for ampicillin $(10 \mu \mathrm{g})$, chloramphenicol $(30 \mu \mathrm{g})$, streptomycin $(10 \mu \mathrm{g})$ (Becton Dickinson, Sparks, Maryland, USA), cotrimoxazole $(25 \mu \mathrm{g})$, tetracycline $(30 \mu \mathrm{g})$, ciprofloxacin $(5 \mu \mathrm{g})$ (Difco Laboratories, Detroit, Michigan, USA), and trimethoprim (Mast Diagnostics Ltd, Bootle, Merseyside, UK) $(5 \mu \mathrm{g})(15,16)$. The antimicrobials were classified as aminoglycosides (STR, AMP), tetracyclines (TE), amphenicols (CHL), fluoroquinolones (CIP), trimethoprim alone (TMP), or in combination with sulfonamides (SXT).

\begin{tabular}{|c|c|c|c|}
\hline Target & Primer Sequences 5'-3' & Amplicon Size, bp & Reference \\
\hline \multirow[t]{3}{*}{ Enteropathogenic E. coli (eae) } & & 422 & $(10)$ \\
\hline & ACACTCCGATTCCTCTGGTG & & \\
\hline & CTTGCACATAAGCAGGCAAA & & \\
\hline \multirow[t]{3}{*}{ Salmonella enterica (invA) } & & 243 & (11) \\
\hline & ACAGTGCTCGTTTACGACCTGAAT & & \\
\hline & AGACGACTGGTACTGATCGATAAT & & \\
\hline \multirow[t]{3}{*}{ Shigella spp.(ipaH) } & & 620 & $(12)$ \\
\hline & GTTCCTTGACCGCCTTTCCGTTACCGTC & & \\
\hline & GCCGGTCAGCCACCCTCTGAGAGTAC & & \\
\hline \multirow[t]{3}{*}{ Vibrio cholera (16S-23S intergenic region) } & & 300 & (13) \\
\hline & AGTCACTTAACCATTCAACCCG & & \\
\hline & TTAAGCGTTTTCGCTGAGAATG & & \\
\hline \multirow[t]{3}{*}{ Class 1 integron (int1) } & & 900 & (14) \\
\hline & TGCGTGTAAATCATCGTCGT & & \\
\hline & CAAGGTTCTGGACAGTTGC & & \\
\hline
\end{tabular}

Table 2. Antimicrobial Susceptibility Testing of the Isolates $\mathrm{a}, \mathrm{b}$

\begin{tabular}{lccccccc}
\hline Bacteria & SXT & TE & TMP & STR & CIP & CHL & AMP \\
\hline EPEC & $28(100)$ & $28(100)$ & $28(100)$ & $28(100)$ & $28(100)$ & $28(100)$ & $28(100)$ \\
S. entarica & $10(27.8)$ & $17(47.2)$ & $10(27.8)$ & $17(47.2)$ & $4(11.1)$ & $1(2.8)$ & $9(25)$ \\
Sh. sonnei & $30(93.7)$ & $28(84.3)$ & $30(93.7)$ & $13(40.6)$ & $1(3.1)$ & $7(18.7)$ & $12(37.5)$ \\
V. cholerae & $23(95.8)$ & $0(0)$ & $23(95.8)$ & $23(95.8)$ & $2(8.3)$ & $22(91.7)$ & $3(12.5)$ \\
Total & $91(75.8)$ & $73(60.8)$ & $91(75.8)$ & $81(67.5)$ & $35(29.2)$ & $58(48.3)$ & $52(43.3)$ \\
\hline
\end{tabular}

a Abbreviations: AMP, aminoglycosides; CHL, amphenicols; CIP, fluoroquinolones; STR,; SXT, sulfonamides; TE, tetracyclines; TMP, trimethoprim alone.

b Data are presented in No. (\%). 
Figure 1. PCR Amplifications Performed in This Study.
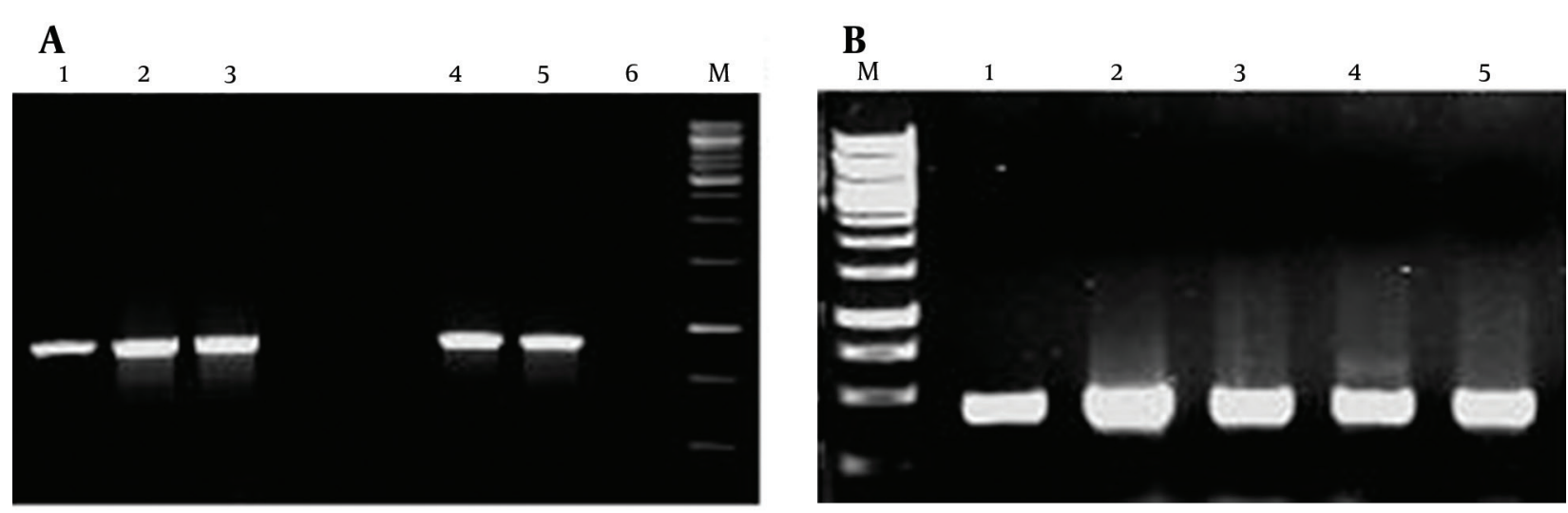

C

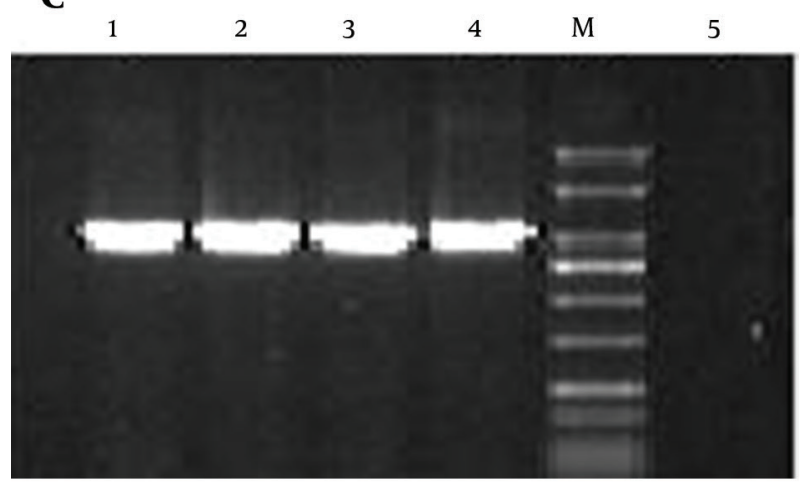

D

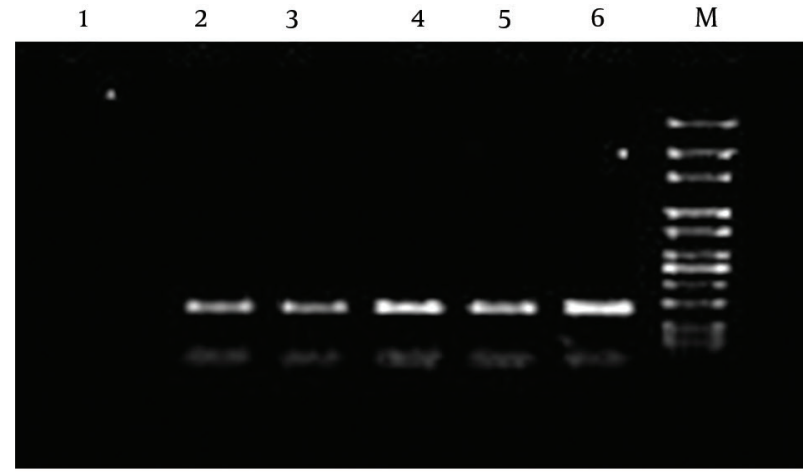

$\mathbf{E}$

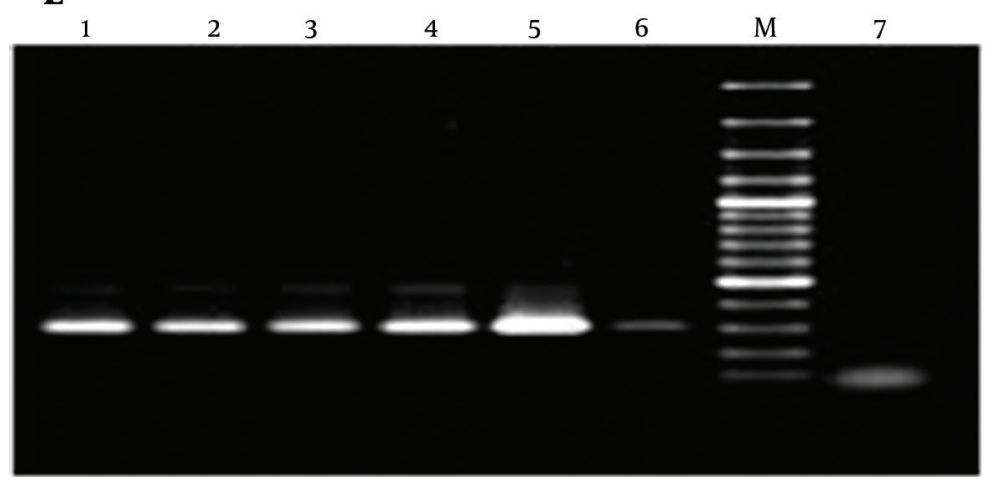

A) PCR amplification of integrase gene (int) of class 1 integron; lanes 1-4: int+ isolates, lane 5: positive control, lane 6: negative control. B) PCR amplification of eae gene; lanes 1-4: eae+ isolates, lane 5: positive control. C) PCR amplification of ipaH gene; lanes 1-3: ipa H+ isolates, lane 4: positive control, lane 5: negative control. D) PCR amplification of hilA gene; lanes 3-6: hilA+ isolates, lane 2: positive control, lane 1: negative control. E) PCR amplification of 16s-23s intergenic spacer region of $V$. cholerae; lanes 2-6: positive isolates, lane 1: positive control, lane 7: negative control.

\subsection{Integron Characterization and Sequencing of Resistance Gene Cassettes}

The total DNA was extracted by boiling method and used as template in the PCR assays for pathogen confirmation and class1 integrons detection. The primer sequences used in this study are shown in Table 1. PCR was performed in a reaction mixture with total volume of 25 $\mu \mathrm{L}$, containing $2.5 \mu \mathrm{L} 10 \mathrm{x}$ Taq polymerase buffer, $0.3 \mu \mathrm{L}$ dNTPs (10 mmol/L), $1 \mathrm{U}$ Taq DNA polymerase, $0.6 \mu \mathrm{L} \mathrm{MgCl}_{2}$ ( $50 \mathrm{mmol} / \mathrm{L}$ ), and $0.3 \mathrm{~mol} / \mathrm{L}$ of each primer. PCR was performed as follows: initial denaturation step at $94^{\circ} \mathrm{C}$ for 5 minutes, followed by 30 cycles consisting of denatur- 
ation $\left(94^{\circ} \mathrm{C}\right.$ for 1 minute), annealing $\left(56^{\circ} \mathrm{C}\right.$ for 1 minute, separately set for each primer pair), and extension $\left(72^{\circ} \mathrm{C}\right.$ for 1 minute), followed by a final extension step at $72^{\circ} \mathrm{C}$ for 3 minutes. One representative of each amplified fragment (eae, ipaH, invA, prVC) was purified using QIAquick gel extraction kit (Qiagen) and subjected to direct sequencing using ABI $3730 X$ capillary sequencer (Genfanavaran, Macrogen, Seoul, Korea). Further analysis of the se quenced fragments was performed using BLAST software.

\section{Results}

\subsection{Clinical Isolates}

A total of 120 enteropathogenic bacteria were included in this study. Twenty eight EPEC, 36 S. enterica, 32 Sh. Son$n e i$, and $24 \mathrm{~V}$. cholerae were randomly selected from our collection, and conventional biochemical tests and PCR analysis confirmed the identity of the isolates.

\subsection{Antimicrobial Susceptibility Analysis}

The highest resistance among the total enteropathogenic bacteria was observed against trimethoprim and cotrimoxazole, especially among EPEC (100\%), Shigella spp (93.7\%) and V. cholerae (95\%) isolates (Table 2). All EPEC were resistant to all tested antibiotics. The foremost resistance profile among Sh. sonnei was SXT/TE/TMP (34.5\%). The most prevalent resistance was seen to trimethoprim and cotrimoxazole (93.7\%) and tetracycline (87.5\%), while only one isolate (3.1\%) was resistant to ciprofloxacin. The most prevalent resistance pattern among $V$. cholerae isolates was SXT/TMP/STR/CHL (33.3\%).

The highest resistance rate was seen to SXT (95\%), streptomycin (95\%), and chloramphenicol (91.7\%), and the least resistance was seen to ciprofloxacin (8.3\%) and ampicillin $(12.5 \%)$, while no resistance was seen to tetracycline. Most of the strains (95\%) were MDR (resistant to $\geq 3$ classes of antimicrobial agents), however, with very unlike combinations of antibiotics. The foremost resistance profile among S. enterica isolates was SXT/TMP/TE/ STR (13.8\%). The most prevalent resistance was seen to streptomycin and tetracycline $(47.2 \%)$, while only $1(2.8 \%)$ isolate was resistant to chloramphenicol (Table 2).

\subsection{Polymerase Chain Reaction Analysis of Class 1 Integrons}

Species- or genus-specific PCR analyses confirmed the identity of the isolates. All 120 isolates were subjected to class 1 integron (int + ) analysis using primers int1-F and int1- $\mathrm{R}$ (5'-conserved region) with an amplification band of $900 \mathrm{bp}$ (Figure 1). The results showed that $16(57.1 \%)$ of 28 EPEC, 9 (25\%) of 36 S. enterica, 13 (40.6\%) of 32 Sh. sonnei, and only $1(4.2 \%)$ of $24 \mathrm{~V}$. cholerae isolates were positive for conserve region of class 1 integron.

\section{Discussion}

The results of this study indicated high prevalence of resistance to cotrimoxazole and trimethoprim among all the studied species. This finding was in agreement with other studies from Europe and Asia $(17,18)$, reporting MDR among members of enterobacterial species, with a high percentage of resistant isolates to cotrimoxazole and trimethoprim. In this study, the observed high resistance to cotrimoxazole and trimethoprim (75.8\%) and streptomycin (67.5\%) suggested probable dominance of the associated resistance gene cassettes within variable region of class 1 integrons, among the isolates.

In this study, class 1 integron was identified in all species and the most prevalence was seen among EPEC isolates (57.5\%), while only one isolate of $V$. cholerae harbored class 1 integron. Twenty five percent of S. enterica and $37.5 \%$ of Sh. sonnei contained class 1 integron, respectively. Recent studies have clearly illustrated the widespread distribution of class 1 integrons among bacterial isolates in Europe (19), the USA, Africa (20) and Asia (21). Emergence of MDR pathogens among Enterobacteriaceae is highly related to integrons, particularly class 1 integrons (22). Reyes et al. showed that class 1 integrons were common (32.4\%) among 191 enterobacterial species (23). In a study by Zhu and colleagues, high prevalence of MDR (95.6\%) was observed among the 90 Shigella strains, isolated from stool of patients in china, and $87.8 \%$ of the MDR strains carried class 1 integrons (3.3\%) (17). According to a study by Ibrahim et al. the prevalence of class 1 integron was $95 \%$ in the outbreak isolates and $97 \%$ in sporadic cases (24). Ibrahim and colleagues reported occurrences of class 1 integron among $40.6 \%$ of EPEC isolated from Sudan (24). In Iran, distribution of class 1 integron in Salmonella isolates in Tehran was 38.1\% (25). This shows an increase in the prevalence of class 1 integron among salmonella isolated of Iranian population.

The data obtained in the present study suggested that class 1 integrons were more widely distributed among members of Enterobacteriaceae than other families of enteropathogenic bacteria including vibrionaceae. In older studies, it was suggested that the integrons were significantly correlated with resistance to certain antibiotics including gentamicin, kanamycin, streptomycin, tobramycin, sulfafurazole, trimethoprim, ampicillin, chloramphenicol, and tetracycline (26). The resistance patterns present in our E. coli, Sh. sonnei, and S. enterica isolates were nearly identical, suggesting that probably these different species have similar mechanisms for attainment of MDR. All of these results together with earlier finding of the extremely efficient interspecies transfer of integron-carrying elements, illustrated that horizontal transfer of integron-carrying elements plays a dominant role in the development of MDR among members of Enterobacteriaceae, independent of species or origin of isolation (10). 
The findings signified the need for monitoring antimicrobial resistance among different members of Enterobacteriaceae, as well as their integron distribution and content. Moreover, further studies are needed to understand the exact mechanisms involved in spreading of integrons among different bacterial genera.

\section{Acknowledgements}

We thank the Research Council of Tarbiat Modares University for supporting the project.

\section{Authors' Contribution}

1- Study concept and design: B. Bakhshi, M. Pourshafie

2-Acquisition of data: N. Eftekhari

3- Analysis and interpretation of data: B. Bakhshi

4- Drafting of the manuscript: B. Bakhshi

5- Critical revision of the manuscript for important intellectual content: B. Bakhshi

6- Statistical analysis: B. Bakhshi, M. Pourshafie

7- Administrative, technical, and material support: B. Bakhshi, M. Pourshafie 8-Study supervision: B. Bakhshi

\section{Financial Disclosure}

This study was supported by a grant from the research council of Tarbiat Modares University.

\section{Funding/Support}

This study was supported by the Research Council of Tarbiat Modares University.

\section{References}

1. Ramana J, Tamanna . dbDiarrhea: the database of pathogen proteins and vaccine antigens from diarrheal pathogens. Infect Genet Evol. 2012;12(8):1647-51.

2. Scarlett TC, Durham RW, Hall IH, Crosswicks RJ, Berkowitz JD, Burnham BS. Synthesis and cytotoxicity of cyanoborane adducts of n6-benzoyladenine and 6-triphenylphosphonylpurine. Met Based Drugs. 2002;9(1-2):19-32.

3. Martinez-Freijo P, Fluit AC, Schmitz FJ, Grek VS, Verhoef J, Jones ME. Class I integrons in Gram-negative isolates from different European hospitals and association with decreased susceptibility to multiple antibiotic compounds. J Antimicrob Chemother. 1998;42(6):689-96.

4. Leverstein-van Hall MA, Box AT, Blok HE, Paauw A, Fluit AC, Verhoef J. Evidence of extensive interspecies transfer of integronmediated antimicrobial resistance genes among multidrugresistant Enterobacteriaceae in a clinical setting. $J$ Infect Dis. 2002;186(1):49-56.

5. van Belkum A, Goessens W, van der Schee C, Lemmens-den Toom $\mathrm{N}$, Vos MC, Cornelissen J, et al. Rapid emergence of ciprofloxacinresistant enterobacteriaceae containing multiple gentamicin resistance-associated integrons in a Dutch hospital. Emerg Infect Dis. 2001;7(5):862-71.

6. Gruteke P, Goessens W, Van Gils J, Peerbooms P, Lemmens-Den Toom N, Van Santen-Verheuvel M, et al. Patterns of resistance associated with integrons, the extended-spectrum beta-lactamase SHV-5 gene, and a multidrug efflux pump of Klebsiella pneumoniae causing a nosocomial outbreak. J Clin Microbiol.
2003;41(3):1161-6.

7. Stokes HW, Hall RM. A novel family of potentially mobile DNA elements encoding site-specific gene-integration functions: integrons. Mol Microbiol.1989;3(12):1669-83.

8. Hall RM, Collis CM. Mobile gene cassettes and integrons: capture and spread of genes by site-specific recombination. Mol Microbiol. 1995;15(4):593-600.

9. Rookey DC.. Integrons, genetic elements responsible for acquisition of antibiotic resistance genes, and their prevalence among gram-negative enterics in poultry litter. uga; 2002.

10. Najibi S, Bakhshi B, Fallahzad S, Pourshafie MR, Katouli M, Sattari $\mathrm{M}$, et al. Distribution of class 1 integrons among enteropathogenic Escherichia coli. Can J Microbiol. 2012;58(5):637-43.

11. Chiu CH, Ou JT. Rapid identification of Salmonella serovars in feces by specific detection of virulence genes, invA and spvC, by an enrichment broth culture-multiplex PCR combination assay.J Clin Microbiol. 1996;34(10):2619-22.

12. Sethabutr O, Venkatesan M, Murphy GS, Eampokalap B, Hoge CW, Echeverria P. Detection of Shigellae and enteroinvasive Escherichia coli by amplification of the invasion plasmid antigen H DNA sequence in patients with dysentery. J Infect Dis. 1993;167(2):458-61.

13. Chun J, Huq A, Colwell RR. Analysis of 16S-23S rRNA intergenic spacer regions of Vibrio cholerae and Vibrio mimicus. Appl Environ Microbiol.1999;65(5):2202-8.

14. Adabi M, Bakhshi B, Goudarzi H, Zahraei SM, Pourshafie MR. Distribution of class I integron and sulfamethoxazole trimethoprim constin in Vibrio cholerae isolated from patients in Iran. Microb Drug Resist. 2009;15(3):179-84.

15. Cockerill FR. Performance standards for antimicrobial susceptibility testing: twenty-first informational supplement.: Clinical and Laboratory Standards Institute (CLSI); 2011.

16. Wikler MA. Performance standards for antimicrobial susceptibility testing: sixteenth informational supplement.: Clinical and Laboratory Standards Institute; 2006.

17. Zhu J, Duan G, Yang H, Fan Q, Xi Y. Multi-drug resistance and characteristic of integrons in Shigella spp. isolated from China. Biomed Environ Sci. 2011;24(1):56-61.

18. Guerra B, Soto S, Cal S, Mendoza MC. Antimicrobial resistance and spread of class 1 integrons among Salmonella serotypes. Antimicrob Agents Chemother. 2000;44(8):2166-9.

19. Leverstein-Van Hall MA, Paauw A, Box AT, Blok HE, Verhoef J, Fluit AC. Presence of integron-associated resistance in the community is widespread and contributes to multidrug resistance in the hospital. JClin Microbiol. 2002;40(8):3038-40.

20. Gassama A, Aidara-Kane A, Chainier D, Denis F, Ploy MC. Integron-associated antibiotic resistance in enteroaggregative and enteroinvasive Escherichia coli. Microb Drug Resist. 2004;10(1):2730.

21. Mathai E, Grape M, Kronvall G. Integrons and multidrug resistance among Escherichia coli causing community-acquired urinary tract infection in southern India. APMIS. 2004;112(3):159-64.

22. Leverstein-van Hall MA, M. Blok HE, T. Donders AR, Paauw A, Fluit AC, Verhoef J. Multidrug resistance among Enterobacteriaceae is strongly associated with the presence of integrons and is independent of species or isolate origin. J Infect Dis. 2003;187(2):251-9.

23. Reyes A, Bello H, Dominguez M, Mella S, Zemelman R, Gonzalez G. Prevalence and types of class 1 integrons in aminoglycosideresistant Enterobacteriaceae from several Chilean hospitals. Antimicrob Chemother. 2003;51(2):317-21.

24. Ibrahim ME, Magzoub MA, Bilal NE, Hamid ME. Distribution of Class I integrons and their effect on the prevalence of multi-drug resistant Escherichia coli clinical isolates from Sudan. Saudi Med J. 2013;34(3):240-7.

25. Ranjbar R, Giammanco GM, Farshad S, Owlia P, Aleo A, Mammina C. Serotypes, antibiotic resistance, and class 1 integrons in Salmonella isolates from pediatric cases of enteritis in Tehran, Iran. Foodborne Pathog Dis. 2011;8(4):547-53.

26. White PA, McIver CJ, Rawlinson WD. Integrons and gene cassettes in the enterobacteriaceae. Antimicrob Agents Chemother. 2001;45(9):2658-61. 